\title{
Personal Service: A Robot that Greets People Individually Based on Observed Behavior Patterns
}

\author{
Dylan F. Glas, Kanae Wada, Masahiro Shiomi, Takayuki Kanda, Hiroshi Ishiguro, Norihiro Hagita \\ ATR Intelligent Robotics and Communication Laboratories \\ Kyoto, Japan \\ (dylan, k_wada, m-shiomi, kanda, hagita)@atr.jp, ishiguro@sys.es.osaka-u.ac.jp
}

\begin{abstract}
We are developing an interactive service robot which provides personal greetings to customers, using a machine-learning approach based on observations of a customer's appearance or behavior from on-board or environmental sensors. For each visit, several features are recorded, such as "time of day" or "number of people in group." A set of classifiers trained by human coders compare the current features with the person's individual history, to determine an appropriate feature for a robot to speak about. This system enables the robot to make context-appropriate comments such as "good morning, you're here very early today." We present the design of our system and an encouraging set of preliminary prediction results based on one month of data taken from real customers at a shopping mall.
\end{abstract}

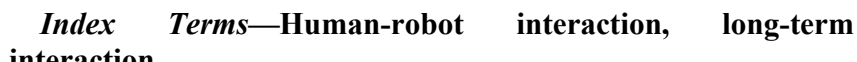
interaction

\section{INTRODUCTION}

In today's high-speed world of technology and massproduction, our daily interactions are beginning to lose the warmth of personal human interactions. What was once an everyday experience of going to a local shop and being greeted personally - "I like your new haircut," or, "You're here late today - did you have a long day at work?" is fading into a nostalgic memory of a lost era. Perhaps this is inevitable our modern world - a cashier working shifts at a busy chain store has little time or inclination to remember hundreds of faces or engage in personal conversations.

Ironically, as the roles of people working in such establishments become more "robotic" in nature, service robots performing similar roles might be able to provide that missing "personal touch". By recording sensor observations of the appearance and behavior of individuals over time in a database, a robot could remember thousands of customers and be able to comment when something is unusual or interesting. We are developing a service robot to greet people with such personal comments, to bring the feeling of personal service back to the experience of daily interactions.

In this report, we present our system design and preliminary results based on one month of customer data collected in a shopping mall.

\section{RELATED WORK}

Long-term interaction and establishing relationships has attracted a lot of attention in human-robot (and human-agent) interaction. For example, Bickmore and Picard studied computer agents that greet users on a daily basis and gradually express a personal relationship [1]. In the Receptionist robot study [2], a series of daily episodes were prepared to engage users into long-term interaction. However, these studies used pre-implemented contents, and they did not introduce techniques for individualizing interaction. Kidd developed a personal robot that conducted long-term interactions with users and tried to establish relationships to help motivate them in a weight loss program [3], but that study involved a closed community of registered users interacting closely with a robot. Our study aims to individualize interaction for a large set of people based only on information from casual, repeated interactions.

\section{IMPLEMENTATION}

\section{A. Environment}

To obtain data about customers, we set up a human tracking system [4] and two pan-tilt-zoom video cameras in the entrance of a shopping mall. The cameras monitored two sets of sliding doors, and their video feeds were processed by a server running OKAO Vision face-recognition software ${ }^{1}$.

\section{B. Data processing}

The basic data processing pipeline that we implemented is shown in Fig. 1. After performing sensor fusion between the face recognition and human tracking data, we grouped sequences of data into discrete "visits" and extracted several features from the sensor data available for each visit. For example, features such as time of day or day of week can be extracted based on visit time. Other features, such as identifying the number of people coming together in a group, can be extracted with some processing of the tracking data.

For each of these features, we also computed time-series statistics based on the person's visit history. For continuous values such as time of day, we computed average, standard deviation, and difference between the current and average values. For discrete values such as day of week or group size, we calculated the mode, relative frequency of the mode, and a Boolean value reflecting whether the current value was equal to the mode. We are still exploring what statistics are useful to include in the learning vector.

\footnotetext{
OKAO Vision, OMRON Corporation.

http://www.omron.com/r_d/coretech/vision/okao.html
} 


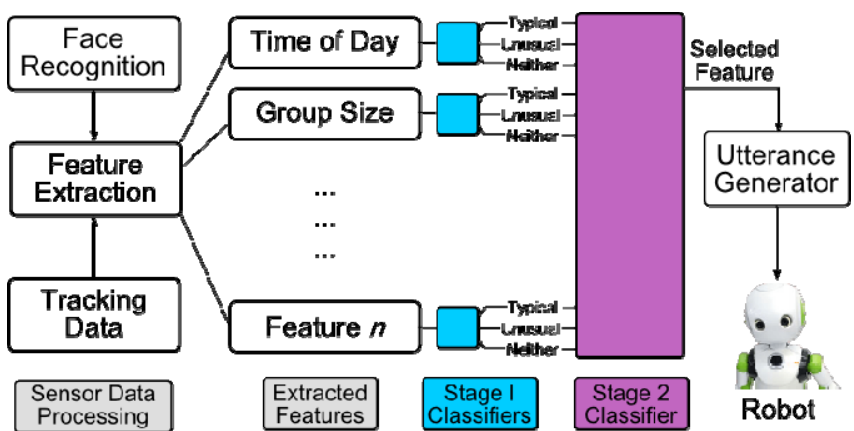

Fig. 1. General summary of the information flow.

\section{Learning}

We divided the learning process into two stages. For each feature, a "Stage I" classifier was trained to identify whether a value could be considered "typical" or "unusual" for that person, based on their history, or whether there was "no pattern" in their behavior. For example, if a customer came between $10 \mathrm{am}$ and $11 \mathrm{am}$ for several days in a row, but at $6 \mathrm{pm}$ on another day, this would be "unusual" value for the "time of day" feature. A "Stage II" classifier was then trained to use the outputs from each Stage I classifier, to determine an appropriate feature for the robot to speak about.

For example, if "time of day" predicted likelihoods of $(50 \%, 25 \%, 25 \%)$ for "typical" "unusual", and "no pattern", but "group size" predicted likelihoods of $(5 \%, 85 \%, 10 \%)$, then it seems likely that it would be more appropriate for the robot to comment on the unusual group size - perhaps the customer brought her grandchild shopping, although she usually comes alone.

Both stages used support vector machine (SVM) classifiers and were trained by data from a human evaluator.

\section{EVALUATION AND RESULTS}

Within one month, we recorded 20 days of video and human position data at the shopping mall, from $10 \mathrm{am}$ to $7 \mathrm{pm}$ on weekdays. Within this data set, we registered individual ID's for 734 individuals, for whom a total of 1278 unique visits were identified.

Table I shows preliminary estimates of the prediction accuracy for features derived from the time of a person's visit. Accuracy was calculated by a "leave-one-out" crossvalidation analysis performed by folding the data sets 5 times.

TABLE I. PREDiction ACCURACY RESUlTS FOR STAGe I

\begin{tabular}{|l|l|}
\hline \multicolumn{1}{|c|}{ Feature } & \multicolumn{1}{c|}{$\begin{array}{c}\text { Prediction accuracy } \\
\text { (from cross-validation) }\end{array}$} \\
\hline Time of day & $78.3 \%$ \\
\hline Day of week & $77.6 \%$ \\
\hline Days since last visit & $76.0 \%$ \\
\hline
\end{tabular}

\section{DISCUSSION}

We consider it quite encouraging that the prediction rates for most of the Stage I learning machines were so high, and we expect that they will be sufficient for reproducing the results of human judgment. After repeating the coding procedure with multiple independent evaluators, we will be able to evaluate whether the prediction accuracy is within the variance of interpersonal variation.

\section{CONCLUSIONS AND FUTURE WORK}

The next step in this research is to conduct a real field trial with a robot in the shopping mall to validate the usefulness of this approach in generating personalized greetings. We will apply the learned classifiers from this study to data from new customers and evaluate their level of satisfaction.

Further in the future, we would like to extend this work to include more complex recognition techniques when they are available, e.g., hairstyle or clothing recognition, or recognizing what objects a person is carrying. As noted earlier, some research into these areas exists but is not stable enough for reliable use in real-world environments.

If successful, this work will provide a valuable proof-ofconcept demonstration of how the unique capabilities of a robot can provide a potentially more personalized interaction experience than a human can. The results of our field study should also give interesting feedback on how real shoppers feel about the "personal touch" of a robot and whether they feel it makes their shopping experience more enjoyable.

\section{ACKNOWLEDGMENT}

We would like to thank Dr. Satoshi Koizumi, Tony Han, and the management of the APiTA Town Keihanna shopping mall for their help in the organization and execution of the data collection. This research was supported by the Ministry of Internal Affairs and Communications of Japan.

\section{REFERENCES}

[1] T. W. Bickmore and R. W. Picard, "Establishing and maintaining long-term human-computer relationships," ACM Transactions on ComputerHuman Interaction (TOCHI), vol. 12, pp. 293-327, 2005.

[2] R. Gockley, A. Bruce, J. Forlizzi, M. Michalowski, A. Mundell, S. Rosenthal, B. Sellner, R. Simmons, K. Snipes, A. C. Schultz, and W. Jue, "Designing robots for long-term social interaction," in Intelligent Robots and Systems, 2005. (IROS 2005). 2005 IEEE/RSJ International Conference on, 2005, pp. 1338-1343.

[3] C. D. Kidd, "Designing for long-term human-robot interaction and application to weight loss," Massachusetts Institute of Technology, 2008.

[4] D. F. Glas, T. Miyashita, H. Ishiguro, and N. Hagita, "Laser-Based Tracking of Human Position and Orientation Using Parametric Shape Modeling," Advanced Robotics, vol. 23, pp. 405428, 2009. 